\title{
Maternal exposure to polycyclic aromatic hydrocarbons diminishes murine ovarian reserve via induction of Harakiri
}

\author{
Andrea Jurisicova, ${ }^{1,2}$ Asako Taniuchi,, ${ }^{1}$ Han Li, ${ }^{1}$ Yuan Shang, ${ }^{1}$ Monica Antenos, ${ }^{3}$ Jacqui Detmar, ${ }^{1}$ \\ Jing Xu, ${ }^{1}$ Tiina Matikainen, ${ }^{4}$ Adalberto Benito Hernández, ${ }^{5}$ Gabriel Nunez, ${ }^{6}$ and Robert F. Casper ${ }^{1,2}$ \\ ${ }^{1}$ Department of Obstetrics and Gynecology, Division of Reproductive Endocrinology and Infertility, and 2Department of Physiology, \\ University of Toronto, Samuel Lunenfeld Research Institute, Mount Sinai Hospital, Toronto, Ontario, Canada. 3Department of Neurobiology and Physiology, \\ Northwestern University, Evanston, Illinois, USA. ${ }^{4}$ Program of Developmental and Reproductive Biology, Biomedicum Helsinki and \\ Hospital for Children and Adolescents, Helsinki University Central Hospital, Helsinki, Finland. ${ }^{5}$ Laboratorio de Nefrología e Inmunología del Transplante, \\ Hospital Universitario Marqués de Valdecilla y Fundación Marqués de Valdecilla-IFIMAV, Santander, Cantabria, Spain. \\ ${ }^{6}$ Department of Pathology, University of Michigan Medical School and Comprehensive Cancer Center, Ann Arbor, Michigan, USA.
}

\begin{abstract}
Maternal smoking during pregnancy is associated with a variety of adverse neonatal outcomes including altered reproductive performance. Herein we provide molecular evidence for a pathway involved in the elimination of the female germline due to prepregnancy and/or lactational exposure to polycyclic aromatic hydrocarbons (PAHs), environmental toxicants found in cigarette smoke. We show that ovaries of offspring born to mice exposed to PAHs contained only a third of the ovarian follicle pool compared with offspring of unexposed female mice. Activation of the cell death pathway in immature follicles of exposed females was mediated by the aryl hydrocarbon receptor (Ahr), as ovarian reserve was fully rescued by maternal cotreatment with the Ahr antagonist, resveratrol, or by inactivation of the $A b r$ gene. Furthermore, in response to PAHs, Ahr-mediated activation of the harakiri, BCL2 interacting protein (contains only BH3 domain), was necessary for execution of cell death. This pathway appeared to be conserved between mouse and human, as xenotransplanted human ovarian cortex exposed to PAHs responded by activation of the identical cell death cascade. Our data indicate that maternal exposure to PAHs prior to pregnancy and/or during lactation compromises ovarian reserve of female offspring, raising the concern about the transgenerational impact of maternal smoking on ovarian function in the human.
\end{abstract}

\section{Introduction}

Polycyclic aromatic hydrocarbons (PAHs) are released into the environment in high quantities as a result of the incomplete combustion of fossil fuels such as wood, coal and petrochemical products. However, a primary route of human exposure to these compounds is cigarette smoke. The adverse effects of PAHs on female reproductive function have been documented in both human epidemiological and animal intervention studies (1-3). Maternal smoking prior to and during pregnancy has many serious developmental consequences manifested both peri-implantation and during gestation (4). Furthermore, smoking dramatically affects the developing fetus, as determined by the high rate of intrauterine growth restriction, perinatal morbidity, sudden infant death syndrome, increased susceptibility to respiratory diseases in infancy, and long-term impairment of cognitive development and behavior (5).

Menopause, characterized by depletion of the resting follicular pool with loss of cyclical ovarian function, marks the end of a woman's reproductive capacity and is accompanied by major changes in female physiology. Both the size of the follicle endow-

Nonstandard abbreviations used: Ahr, aryl hydrocarbon receptor; BaP, benzo(a)pyrene; BPDE, BaP diol epoxide; DMBA, 7,12-dimethylbenz(a)anthracene; $\mathrm{Hrk}$, harakiri, BCL2 interacting protein (contains only BH3 domain); $\alpha$-NF, $\alpha$-naphthoflavone; $\mathrm{PAH}$, polycyclic aromatic hydrocarbon; XRE, xenobiotic response element. Conflict of interest: The authors have declared that no conflict of interest exists. Citation for this article: J. Clin. Invest. 117:3971-3978 (2007). doi:10.1172/JCI28493. ment at birth as well as accelerated germ cell loss during life can effect the age at which menopause occurs. One of the most consistent findings in the epidemiological literature is a link between cigarette smoking and onset of early menopause (reviewed in refs. 6,7$)$. Thus it is not surprising that direct exposure to cigarette smoke or PAHs results in accelerated loss of primordial follicles, leading to premature exhaustion of the ovarian pool $(8,9)$. Indeed, studies conducted in animal models demonstrated that females born to mothers exposed to cigarette smoke or PAHs during pregnancy had a significantly decreased number of ovarian primordial follicles $(10,11)$. These results complement the observation of reduced fecundity in women prenatally exposed to maternal cigarette smoke (12).

There are several possible pathways triggered by PAHs that can lead to changes in cellular function. These compounds are capable of directly altering DNA structure via formation of DNA adducts (13). In addition, both 7,12-dimethylbenz(a)anthracene (DMBA) and benzo(a)pyrene (BaP), 2 prototypical PAHs, modulate activity of the aryl hydrocarbon receptor (Ahr), a member of the basic helix-loop-helix family of transcription factors (14). Upon receptor activation facilitated by ligand binding, the Ahr translocates to the nucleus, where it alters the expression of target genes via binding to specific DNA sequences designated as xenobiotic response elements (XREs). The expression of a variety of genes has been reported to be affected by activated Ahr, including xenobiotic metabolizing enzymes, growth factors, cytokines, and other transcription factors (15). 
A

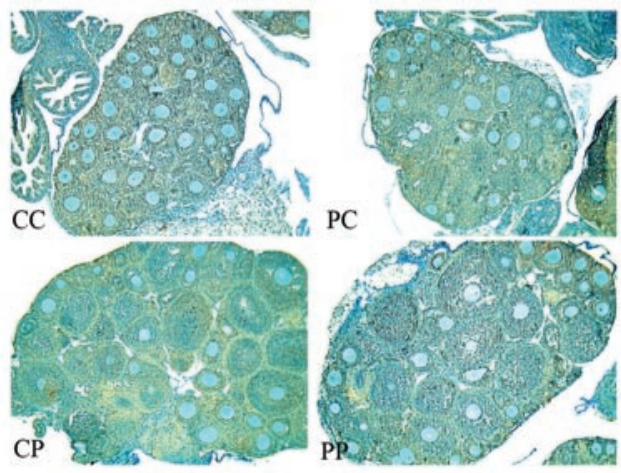

B
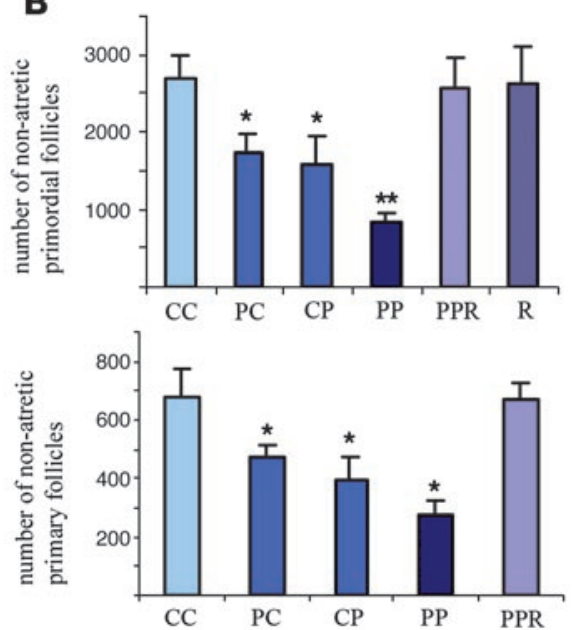

Figure 1

Effect of maternal treatment with PAHs on the ovarian follicle endowment in female offspring. (A) Morphological appearance of ovaries from 3-week-old female daughters of mothers exposed to vehicle only (CC), PAH exposure (cumulative dose of $6 \mathrm{mg} / \mathrm{kg}$ ) before pregnancy (PC), $\mathrm{PAH}$ exposure (cumulative dose of $6 \mathrm{mg} / \mathrm{kg}$ ) during lactation (CP), and PAH exposure (cumulative dose of $12 \mathrm{mg} / \mathrm{kg}$ ) both before pregnancy and during lactation exposure (PP). (B) Quantitative representation of the morphometric analysis of the ovarian pool for nonapoptotic oocytecontaining primordial (resting) and primary (growing) follicles in different treatment groups is expressed as mean \pm SEM. Each treatment group shown represents combined data obtained from at least 2 female offspring per litter from each treated mother: $\mathrm{CC}, n=11 ; \mathrm{PC}, n=18$; $\mathrm{CP}, n=11$; PP, $n=15$. Effectiveness of AHR antagonist was assessed in mothers from the PP group supplemented with resveratrol (PPR), or mothers from the CC group (vehicle) supplemented with resveratrol (R) (cumulative dose of $120 \mathrm{mg} / \mathrm{kg}$ ). Two to three females per litter from each mother were subjected to morphometric analysis (R, $n=6$; PPR, $n=9)$. ${ }^{*} P<0.05$ relative to control; ${ }^{* *} P<0.05$ relative to all treatment groups.

Ahr ligands also activate cell death pathways. Both human and murine ovaries respond to $\mathrm{PAH}$ exposure by induction of apoptosis of primordial follicles in an Ahr-dependent manner (16). PAHengaged Ahr complexes induce expression of the proapoptotic Bcl-2 family member Bax, the activity of which is necessary for the initiation of cell death in resting oocytes $(11,16)$. The purpose of the present study was to investigate the adverse gonadal effects of prepregnancy and lactational exposure of female progeny to PAHs and to explore the possible mechanisms involved. We identified the harakiri, BCL2 interacting protein (contains only $\mathrm{BH} 3$ domain) (Hrk) as one of the cell death genes involved in the regulation of the follicular pool downstream of Ahr activation. Interference with this pathway by maternal exposure to resveratrol could prevent the oocyte depletion induced by PAHs via suppression of Hrk expression. This pathway appears to be conserved, as human ovarian cortical xenotransplants responded similarly to murine ovaries.

\section{Results}

Since most women stop smoking after confirmation of pregnancy, we designed an animal model that would avoid direct exposure of fetuses to the studied compounds and thus more closely reflect human exposure. To mimic exposure in human smokers, a mixture of relatively low doses of 2 prototypical PAHs (DMBA and $\mathrm{BaP}$ ) were given subcutaneously to adult female mice before pregnancy and/or during lactation. There was no direct treatment during pregnancy. Based on the published concentration of total PAHs found in cigarettes (17), the total dose accumulated by the mice during the 3-week period of treatment was estimated to be equivalent to approximately 25 packs of cigarettes, consistent with potential exposure in a heavy smoker.
PAH treatment of female mice prior to conception or during lactation did not cause any differences in the litter size at birth or at weaning. The biochemical response in the mother as well as in the offspring was monitored by the induction of the prototypical $\mathrm{PAH}$-induced Ahr target gene, Cyp1A1, in the lung. There was a significant increase in the Cyp1A1 protein levels in both offspring and dams reflecting the dose and mode of exposure (data not shown). The biological impact of treatment was revealed when the ovarian follicular pool in the offspring, sacrificed just prior to the onset of puberty, was assessed. Female offspring of mothers exposed to PAHs either prior to pregnancy or during lactation contained a significantly reduced resting (primordial) and early growing (primary) follicle pool. Further follicular depletion was observed with combined prepregnancy and lactational exposure, as offspring of exposed females retained only $30 \%$ of the control follicular dowry (Figure 1, A and B).

Previous reports have established that $\mathrm{PAH}$-induced destruction of primordial follicles in vitro is mediated by Bax and this adverse effect could be blocked by simultaneous treatment with the synthetic Ahr antagonist, $\alpha$-naphthoflavone ( $\alpha$-NF; ref. 16). Thus we next investigated whether an Ahr antagonist, administered in vivo to the exposed mothers, could block the PAH-induced follicle depletion observed in the daughters. We used resveratrol, a competitive inhibitor of Ahr, since we had previously demonstrated that resveratrol is a pure Ahr antagonist with no agonistic activity (18). Biweekly maternal cotreatment with resveratrol in addition to PAHs completely prevented depletion of the primordial and primary follicles in the female offspring, while resveratrol alone had no significant effect on the quiescent follicle pool (Figure 1B).

Exposure to PAHs had been previously linked to formation of DNA adducts, which could secondarily lead to cell death through 
A

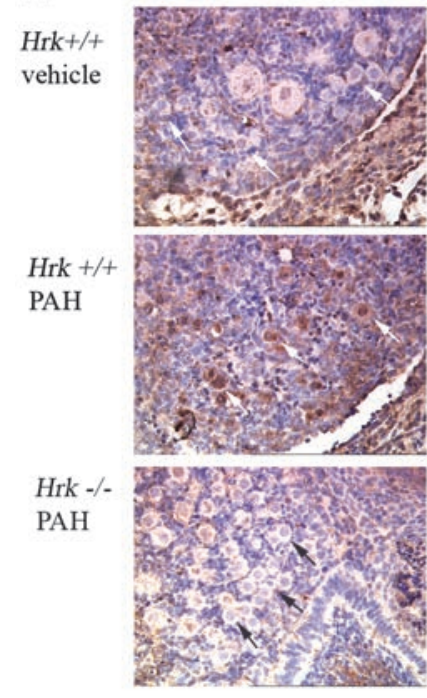

B

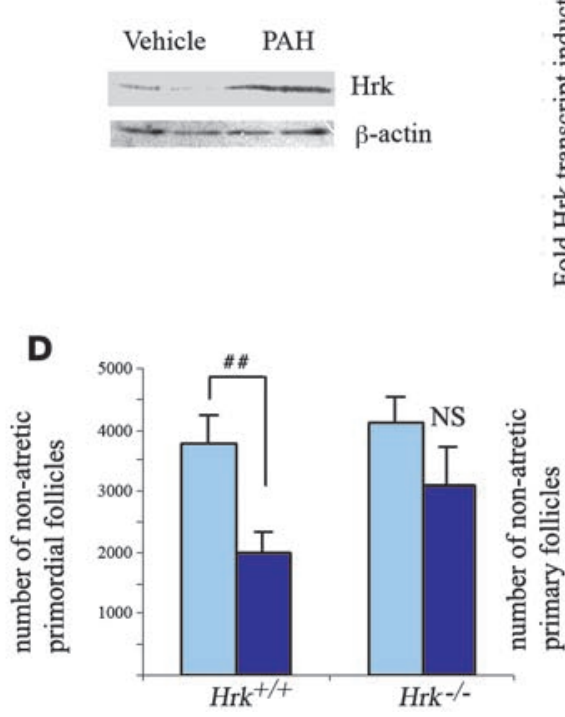

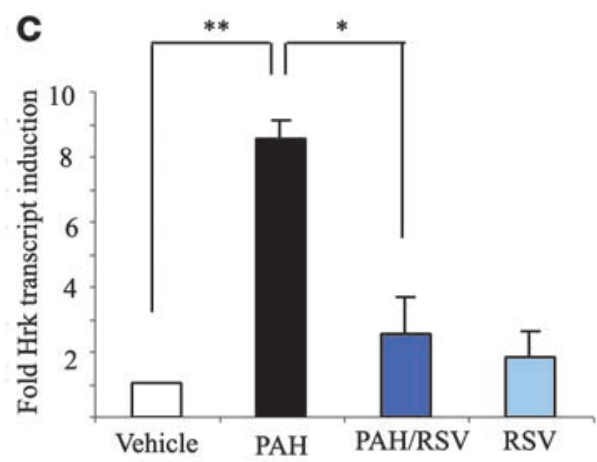

Vehicle

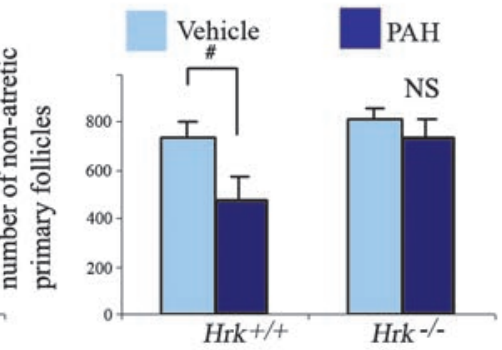

\section{Figure 2}

PAH-driven Hrk induction and its functional requirement in regulation of ovarian pool. (A) Immunohistochemical analysis of Hrk protein (brown staining) in neonatal mouse ovaries following 24 hours of exposure to vehicle or $1 \mu \mathrm{M} D \mathrm{DBA} / \mathrm{BaP}$ each during in vitro culture. Induction was observed in oocytes and granulosa cells of both primordial (black and white arrows) and primary follicles. Magnification, $\times 400$. Hrk-deficient ovaries did not show upregulation of immunoreactivity in oocytes after PAH exposure, confirming the specificity of antibody. (B) Western blot analysis of neonate ovaries under the same conditions as described above. Upon adjustment to $\beta$-actin ratio, Hrk protein, migrating at approximately $15 \mathrm{kDa}$, was induced approximately 2.5 -fold $(P=0.03)$. (C) Induction of Hrk transcript in in vitro-treated neonatal ovaries 24 hours after exposure to vehicle, $1 \mu \mathrm{M}$ DMBA/BaP each (PAHs), treatment with $\mathrm{PAH}$ and $2 \mu \mathrm{M}$ resveratrol (PAH/RSV), or $2 \mu \mathrm{M}$ resveratrol alone (RSV). The expression data are shown as mean \pm SEM of fold change in comparison with the vehicle-treated group and were obtained from 5 sets of independent ovaries per treatment. PAH induced significant $\left({ }^{* *} P=0.0006\right)$ upregulation of Hrk transcript, which was repressed by resveratrol treatment $\left({ }^{\star} P=0.005\right)$. RSV or RSV/PAH groups were not significantly different from vehicle-treated ovaries. (D) Ovaries from Hrk-deficient females were protected from $\mathrm{PAH}$-induced depletion of primordial and primary follicles. Numerical representation of morphometric analysis of ovarian pool for nonapoptotic oocyte-containing primordial (resting) and primary (growing) follicles in sibling offspring born to heterozygote mothers exposed to PAHs prior to pregnancy and during lactation. Data are expressed as mean number of primordial and primary follicles \pm SEM. Each genotype group represents combined data of offspring born to mothers exposed to vehicle $\left(\mathrm{Hrk}^{+/+}, n=5 ; \mathrm{Hrk}^{-1-}, n=7\right)$ or treated with $\mathrm{PAH}\left(\mathrm{Hrk}^{+/+}, n=4 ; \mathrm{Hrk}{ }^{-/}, n=5\right)$. A significant reduction of follicles was only observed in the wild-type ovaries ( ${ }^{\prime} P=0.03$; $\# P=0.05$; NS, not significantly different).

interference with basic cellular functions (19). In order to establish whether DNA adduct formation was contributing to elimination of ovarian follicles in our model, we used immunocytochemical analysis of $\mathrm{BaP}$ diol epoxide (BPDE) nuclear staining, reflecting DNA adduct formation (20) in PAH-exposed ovarian tissue. However, no difference could be observed between control and PAHexposed groups in the number of primordial and primary follicles with and without a BPDE nuclear signal (data not shown).

Follicle loss, whether induced or spontaneous, has been linked to activation of cell death via apoptotic mechanisms. A subset of cell death regulatory pathways in mouse germ cells have been previously described (3). However, the involvement of various $\mathrm{BH} 3$ only family members in regulation of the ovarian pool size is currently unknown. Several recent publications reported that Hrk (21) is upregulated in cells of the immune system upon exposure to dioxin $(22,23)$, a synthetic ligand known to specifically target Ahr. In order to determine whether PAHs are capable of activating Hrk expression in ovarian tissue, we exposed postnatal day 4 ovaries, enriched in primordial and primary follicles, in vitro to PAHs. Real-time RT-PCR revealed that within 24 hours of PAH exposure, ovarian tissue responded by an approximately 6-fold increase in the expression of the Hrk transcript when compared with vehicle-treated tissue (Figure $2 \mathrm{C}$ ). Cotreatment of ovaries with either resveratrol (Figure 2C) or $\alpha$-NF (Supplemental Figure 1; supplemental material available online with this article; doi:10.1172/JCI28493DS1) prevented induction of Hrk, suggesting that resveratrol may exert its rescuing effect at least in part by inhibiting Hrk expression. Immunocytochemical analysis confirmed accumulation of Hrk protein in both primordial and primary oocytes as well as in the granulosa cells (Figure 2A), and this expression remained elevated for a period of 48 hours. Western blot analysis of postnatal day 4 ovaries exposed to PAHs in vitro (Figure $2 \mathrm{~B})$ revealed approximately a 2.5 -fold increase $(P=0.03)$ in Hrk protein expression.

While these observations indicate that PAH-induced cell death is preceded by Hrk upregulation, this relationship may be purely correlative. Therefore, we next examined whether disruption of Hrk provides protection from PAH-induced follicle death in vivo. We used $\mathrm{Hrk}^{+/-}$mothers treated with PAHs prior to pregnancy and during lactation, as this treatment had resulted in the most dramatic depletion of follicles (see Figure 1B). Upon mating to $\mathrm{Hrk}^{+/-}$ males, the resulting offspring were genotyped and the ovarian 
A
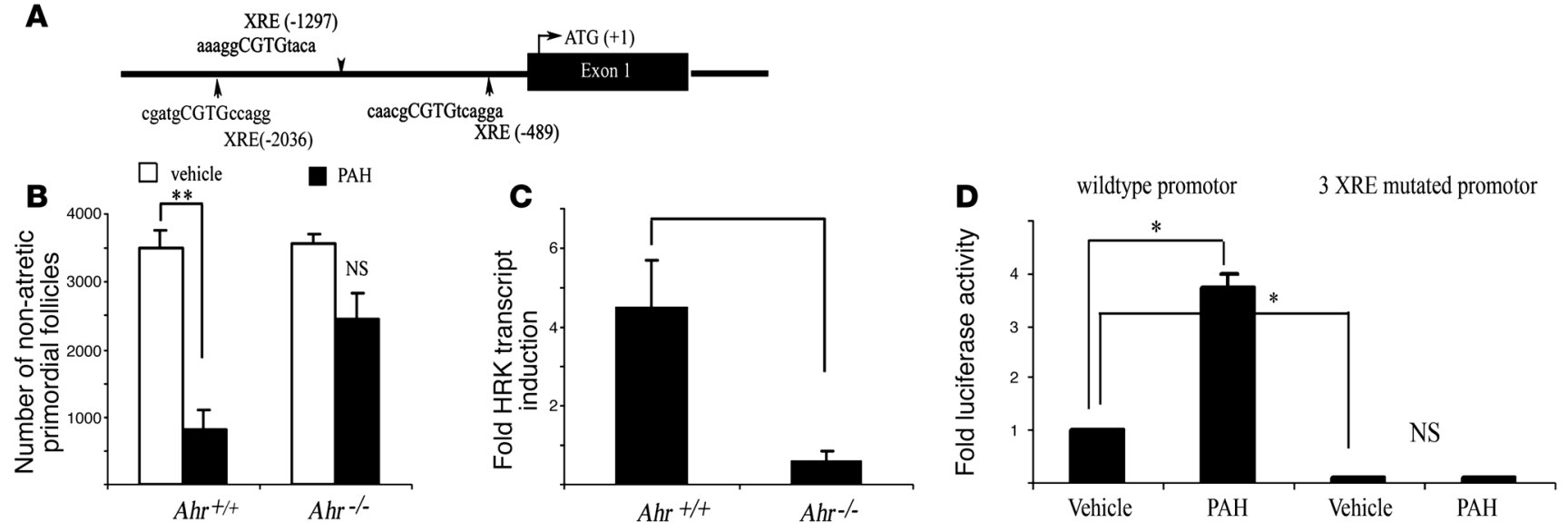

Figure 3

Ahr is involved in in vivo-induced follicle destruction in female offspring after maternal exposure to PAHs. (A) Schematic outline of putative XRE elements, identified by a MatInspector computer algorithm within 5,000 bp of murine Hrk promoter. (B) Ahr-deficient offspring born to mothers exposed to PAHs prior to pregnancy and during lactation retained a significantly larger primordial follicle pool than their wild-type sibling sisters. Quantitative representation of the morphometric analysis of the ovarian pool for nonapoptotic oocyte-containing primordial follicles is expressed as mean \pm SEM. Each genotype group represents combined data from offspring born to mothers exposed to vehicle $\left(A h r^{+/+}, n=4 ; A h r^{-1-}, n=3\right)$ or treated with $\mathrm{PAH}\left(A h r^{+/+}, n=7 ; A h r^{-1-}, n=3\right)$. A significant reduction of follicles was only observed in the wild-type ovaries $\left({ }^{* *} P=0.0003\right)$. (C) $A h r-$ deficient neonatal ovaries failed to upregulate Hrk transcript expression in response to PAHs over a 24-hour period. The expression data are shown as fold change in $\mathrm{PAH}$-treated ovary compared with vehicle-treated ovary from the same female (mean $\pm \mathrm{SEM}$ ) and were obtained from 4 sets of independent ovaries per treatment/genotype. PAH induced a significantly different response in wild-type and $A h r$-deficient ovaries $(P=0.007)$. (D) PAH treatment ( $1 \mu \mathrm{M}$ DMBA/BaP each) induces expression of luciferase driven by Hrk promoter in transient transfection assay using HEK cells $\left({ }^{*} P<0.01\right)$. Activity of the promoter was greatly reduced by mutation of all $3 \mathrm{XRE}$ sites in the presence of vehicle, but no induction occurred after $\mathrm{PAH}$ exposure using mutated construct. Data are shown as mean $\pm \mathrm{SEM}$ of 3 independent experiments performed in pentuplicate and are expressed as fold change of luciferase/ $\beta$-galactosidase ratio.

follicular pools of wild-type and Hrk-deficient sisters compared. Follicle counts revealed partial protection of primordial follicles and almost complete protection of primary follicles in female offspring lacking the Hrk gene (Figure 2C). Our results therefore indicate that Hrk is required for the in vivo primary follicle loss caused by maternal PAH exposure.

Since it appeared that $\mathrm{PAH}$-induced follicle destruction required transcriptional upregulation of Hrk, we further explored the possibility that Hrk may be an Ahr-regulated cell death gene. Results of computer-based scanning of the 5,000-bp sequence upstream of exon 1 of murine Hrk gene using the MatInspector (http://www. genomatix.de/products/MatInspector/) algorithm identified 3 putative XREs (Figure 3A).

In order to establish whether Ahr is necessary for the observed in vivo destruction of follicles resulting from maternal exposure to PAHs, we treated $\mathrm{Abr}^{+/-}$mothers prior to pregnancy and during lactation and compared the follicle profile in their daughters at the time of weaning. As predicted, wild-type offspring lost a substantial amount of their resting ovarian follicular pool. In contrast, Abr-deficient sisters were not significantly affected (Figure 3B). This observation is consistent with previous reports of Ahr-mediated follicle depletion during in vitro PAH exposure (16). To determine whether Ahr is required for Hrk expression, we exposed neonatal ovaries from females lacking the Abr gene or their wild-type siblings to PAHs for 24 hours in vitro and then analyzed Hrk expression. While wild-type ovaries induced Hrk transcript 24 hours after PAH exposure, ovaries from females lacking $A h r$ remained unresponsive (Figure 3C). This observation indicates that PAH-mediated expression of Hrk in ovaries depends on Ahr activity. As the Hrk promot- er contains 3 putative XRE sites, it is possible that Hrk is directly regulated by AHR. Using a luciferase reporter driven by Hrk promoter, we detected an approximately 3.7-fold increase in luciferase activity triggered by PAH exposure (Figure 3D). Almost no detectable luciferase activity was recorded with or without PAH exposure when we used the $\Delta 70 H r k$ promoter, missing the last $70 \mathrm{bp}$ adjacent to exon 1 (data not shown). Using site-directed mutagenesis, we modified all 3 of the putative XRE sites in the wild-type Hrk promoter. These mutations totally abolished the increase in luciferase activity caused by PAH exposure (Figure 3D), indicating that Hrk is directly regulated by AHR.

We next decided to assess whether Bax is also upregulated in primordial and primary follicles in ovaries of $\mathrm{PAH}$-exposed offspring. Consistent with previous reports, Bax staining in both follicle types increased, indicating accumulation of Bax protein (Figure 4A). Since we had observed that females lacking Hrk are protected from follicle loss, we also wished to determine whether ovaries of Hrk-deficient daughters upregulate Bax expression in response to the PAH exposure. Immunocytochemistry indicated no change in the immunoreactivity for Bax in both primordial and primary follicles of $\mathrm{PAH}-$ exposed and vehicle-treated Hrk-deficient females (Figure 4A). In order to confirm failure of Bax induction, we analyzed Hrk-deficient neonatal ovaries exposed to $\mathrm{PAH}$ in vitro for induction of $\mathrm{Bax}$ transcript. Interestingly, while wild-type ovaries upregulated Bax transcript as previously reported (16), Hrk-deficient ovaries did not respond with induction of Bax transcript (Figure 4B).

Finally, we explored whether the PAH-induced Hrk pathway is active in human follicles as well. Previously, we described that human ovarian cortical xenografts, transplanted subcutaneously 

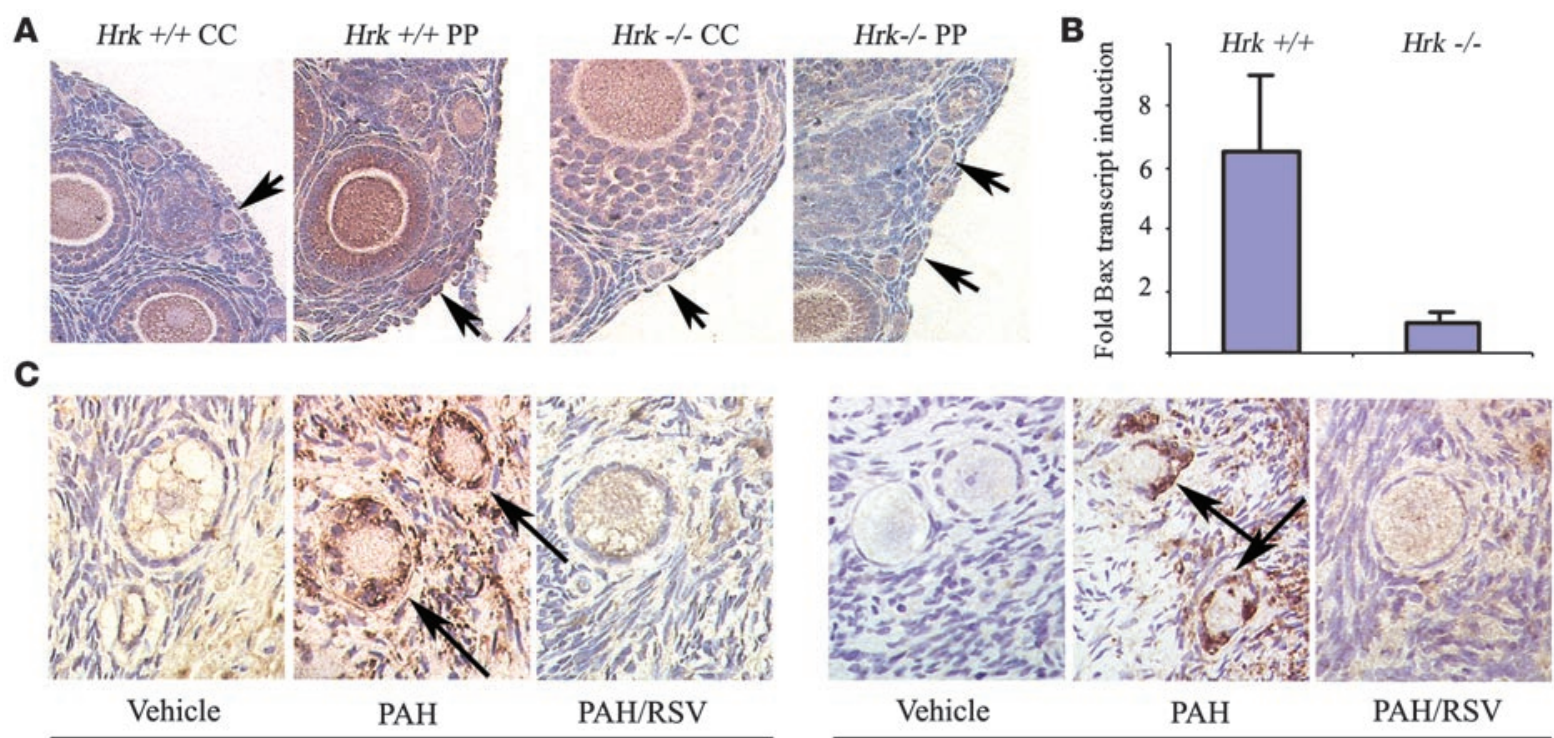

Set 1

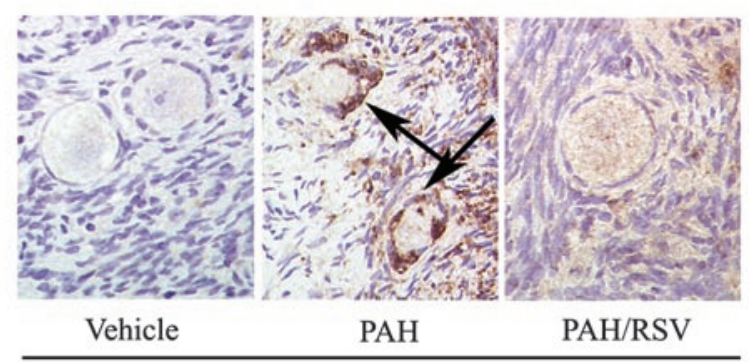

Set 2

\section{Figure 4}

Regulation of Hrk expression in Bax-deficient mouse ovaries and in human ovarian cortical strips. (A) Induction of Bax expression in ovaries of 3-week-old littermates exposed to vehicle only or PAH treatment prior to pregnancy and during lactation. While Bax induction was evident in both primordial and primary follicles (arrows) in wild-type ovaries upon PAH exposure, induction was not evident in the Hrk-deficient females. Note that larger follicles with several layers of granulosa cells also abundantly expressed Bax. However, this expression was regulated by gonadotropins and was independent of PAH treatment. Images are representative results of at least 3 different animals/genotype/treatment. (B) Hrk-deficient neonatal ovaries failed to upregulate Bax transcript expression in response to in vitro exposure to PAHs over a 24-hour period. The expression data are shown as fold change in PAH-treated versus vehicle-treated ovary from the same female (mean \pm SEM) and were obtained from 7 sets of independent ovaries per treatment/genotype. PAH induced a significantly different response between wild-type and $H r k$-deficient ovaries $(P=0.035)$. (C) PAHs induce Hrk expression in human primary (set 1) and primordial (set 2) follicles in ex vivo model. Representative images of immunohistochemical analysis of Hrk protein levels, evidenced by brown staining, in follicles of ovarian grafts 48 hours after exposure to vehicle, $\mathrm{PAH}$, or $\mathrm{PAH}$ and resveratrol. Follicles exposed to PAHs have abnormal shrinking morphology typical of degenerating apoptotic follicles. Induction of Hrk was observed primarily in the granulosa cells (arrows) as well as in the surrounding stroma and was almost completely abolished in both cell types by cotreatment with resveratrol (magnification, $\times 1,000$ ). Data shown are representative results observed from 2 independent sets of experiments using tissues from 2 different patients. No immunoreactivity was observed when primary antibody was omitted (data not shown).

into immunodeficient mice and subsequently exposed to PAHs, activated cell death and upregulated Bax in a manner similar to that of mouse ovarian tissue (16). Thus we used the same approach and examined expression of Hrk in human ovarian cortical xenografts removed from mice exposed to vehicle, PAHs, or PAHs plus resveratrol. Immunohistochemistry revealed induction of Hrk protein, particularly in the granulosa cells of follicles, as well as in the surrounding stroma (Figure 4C). This induction was suppressed by cotreatment with resveratrol, indicating conservation of the PAHAHR-Hrk molecular pathway between rodents and humans.

\section{Discussion}

The loss of primordial and primary follicles has profound biological consequences. Despite controversial studies that suggest existence of putative oogonial stem cells $(24,25)$, it is generally accepted that mammals are born with a finite number of primordial follicles that are incapable of proliferating with advanced maternal age. The loss of oocytes over time appears to be irreversible, as in many model systems depletion of the resting ovarian follicle pool eventually results in sterility $(26,27)$. Previous studies in animal models have clearly demonstrated the ovotoxic effect of direct exposure to PAHs, targeting mainly resting and early growing follicles $(8,9,28-30)$, although it is not possible to exclude that other cell types contribute to follicle demise. Limited data are available regarding the effects of maternal exposure to these compounds on the follicular dowry of the offspring. Since BaP and DMBA are known PAH components of cigarette smoke, our results raise the possibility that human smokers, even if they stop smoking during pregnancy, may have offspring with a decreased number of oocytes, potentially leading to abnormal ovarian function, decreased fertility, and/or early menopause. This suggestion is further supported by epidemiological evidence that women with prenatal exposure to maternal cigarette smoking have reduced fecundity (12).

While a large volume of information exists regarding the toxic effects of PAH, comparatively little is known about the mechanisms by which these compounds exert their cytotoxic effects. Both human and murine ovaries respond to PAH exposure by activation of cell death within oocytes of resting follicles, followed by granulosa cell demise, in an Ahr-dependent manner (16). The $\mathrm{PAH}$-activated Ahr complex transcriptionally regulates the proapoptotic Bcl-2 family member Bax, expression of which appears to be necessary for the induction of cell death in primordial follicles, since animals lacking Bax are almost completely resistant to the loss of this follicle type $(11,16)$

There are likely several parallel mechanisms that result in PAHinduced ovarian toxicity, but downstream effector genes required for the cellular commitment to death remain obscure. Here we describe what we believe is a novel Hrk-driven death pathway par- 
ticularly relevant to destruction of primary follicles. The expression of Hrk appears to be regulated by Ahr, since ovaries from $\mathrm{Abr}$ deficient females do not upregulate Hrk in response to PAHs and mutation of all 3 XRE sites in the Hrk promoter abolished promoter activity triggered by PAHs. We also observed that mutations in the XRE greatly diminished luciferase activity in the presence of vehicle. This is likely caused by the presence of endogenous ligands or contaminating dioxin-like compounds in serum, as these Ahr agonists also require functional XREs for their response. Furthermore, resveratrol, a naturally occurring Ahr antagonist, produced by spermatophyte plants and found in some red wines, is capable of preventing follicular loss triggered by PAHs in the offspring of exposed mothers. In addition, this pathway appears to be conserved, as human ovarian cortical tissue responded to PAHs by induction of HRK likely by AHR-mediated action. Using MatInspector algorithm, we have identified at least 5 putative XREs in the human HRK promoter (data not shown).

Hrk was originally cloned as a $\mathrm{Bcl}-2-$ and $\mathrm{Bcl}-\mathrm{x}$-interacting protein (21) that facilitates cell death via sequestering these antiapoptotic partners from multichannel death-inducing molecules such as Bax (31). In several model systems, particularly in neuronal (32-34) and immune system cells (35), induction of Hrk expression is accompanied by cell death. However, only 3 reports have addressed the functional importance of Hrk expression in cell death (36-38). While Hrk does not bind to Bax (21), it is clear that, at least in neurons, Bax operates downstream of Hrk, forming pores in the outer mitochondrial membrane and allowing release of apoptotic factors sequestered within the mitochondria (33). These studies also showed that Hrk is transcriptionally upregulated in Bax-deficient neurons upon administration of cell death stimuli, even though these cells are protected from apoptosis (33). Based on our results, it is apparent that disruption of Hrk is sufficient to abolish depletion of early growing primary follicles induced by PAHs, indicating that at least at this stage there is no redundancy with Bax. Alternatively, Hrk may work as a sensor upstream of Bax, as previously proposed for other BH3-only proteins (39). In contrast to Bax, death of the quiescent pool of oocytes at the primordial stage was only partially prevented by Hrk deficiency. This finding suggests that, in primordial follicles, activation of other cell death genes working upstream of Bax is needed for execution of cell death and that Hrk aids, but is not totally responsible for, the commitment to death. The partial protection of primordial follicles cannot be due to a transcriptional increase of Bax in Hrk-deficient follicles, as evidenced by the lack of change in immunoreactivity of Bax protein and lack of Bax transcript induction upon PAH exposure. However, the primordial follicle protection from death may still be linked to posttranslational modification of Bax required for the death-inducing activity of this protein (40). It is also possible that another BH3 family member can be activated in primordial follicles in response to PAHs and can facilitate Bax killing, since a high degree of functional redundancy has been reported for several members of the BH3-only gene family $(39,41)$. Therefore, the BH3-only and Bax family members act in concert to tilt the balance in the cell toward the apoptotic program, not only in a cell type-specific manner but also in a developmental stage-specific (e.g., resting versus early growing oocyte pool) manner.

The results of our studies demonstrate that the exposed fetal and neonatal germ lineage is very sensitive to PAH compounds transmitted from the mother to the progeny. To our knowledge, this is the first animal study showing that maternal exposure to
PAHs prior to conception and during lactation could cause ovotoxicity in the next generation. Furthermore, we created a valuable in vivo animal model that could be used to examine the long-term reproductive effects of other environmental toxicants, for example, polycyclic chlorinated biphenyls, which have also been reported to be transmitted transplacentally or through breast milk (42).

As we observed substantial reproductive risks in female progeny of exposed dams, one has to consider the clinical implications of chronic exposure of woman to environmental chemicals, especially cigarette smoke PAHs. Recent healthcare campaigns promote antismoking interventions during pregnancy as a high priority in prenatal care. However, even though most women might stop smoking once a pregnancy is detected, transplacental exposure of the fetus to PAHs, which could accumulate in several tissues, especially body fat, pose a significant biohazard to the developing baby. Moreover, these compounds may also cause epigenetic changes in the ovarian tissue, which could contribute to transgenerational effects such as those observed in this study. Unfortunately, exposure to PAHs from secondhand smoke can be difficult to prevent. Attempts to reduce exposure to these environmental pollutants have not been successful, nor are they likely to be until internal combustion engines, the burning of fossil fuels, and cigarette smoking are eliminated. Therefore, we believe future research should be aimed at developing methods to antagonize the adverse effects of toxic Ahr ligands as one step toward the improvement of human health, including benefits for long-term fetal development and well-being.

\section{Methods}

Animals and treatments. Eight- to ten-week-old female and male inbred C57BL/ 6 mice were obtained from The Jackson Laboratory, housed in plastic cages, provided food and water ad libitum, and maintained on a 12-hour light/12-hour dark cycle at a controlled temperature of about $22^{\circ} \mathrm{C}$. The animals were allowed to acclimate to the animal facilities for 2 weeks prior to the initiation of treatment. BaP and DMBA (Sigma-Aldrich) were freshly prepared in corn oil before each treatment. Animals were divided into subgroups consisting of 5 female mice, as described below. The preconception treatment was given subcutaneously to mice once a week for 3 weeks, and those mice were then mixed with age-matched male mice for mating. There was no treatment during gestation. The maternal lactational treatments were given on the third day after giving birth and then once a week for 3 weeks. Group 1 (vehicle control) was given corn oil $(2.5 \mathrm{ml} / \mathrm{kg}$ ) before pregnancy and during breast-feeding. Group 2 was administered BaP/DMBA ( $1 \mathrm{mg} / \mathrm{kg}$ each per week) before pregnancy and corn oil during lactation. Group 3 received corn oil before pregnancy but was injected with $\mathrm{BaP} / \mathrm{DMBA}$ during breastfeeding. Group 4 was treated with $\mathrm{BaP} / \mathrm{DMBA}$ both before pregnancy and during lactation. For experiments with resveratrol cotreatment, females were injected twice weekly with $10 \mathrm{mg} / \mathrm{kg}$ resveratrol (Sigma-Aldrich) dissolved in propylene glycol, in addition to the weekly $\mathrm{BaP} / \mathrm{DMBA}$ treatment. There was no treatment during pregnancy. Female offspring of treated dams were sacrificed at approximately 3 weeks of age, between postnatal days 24-26.

Mice carrying an Hrk- or Abr-deficient allele on the C57BL/ 6 genetic background were genotyped by PCR as previously described $(37,43)$. Heterozygote females were subjected to identical treatment as groups 1 and 4 and mated to heterozygote males of appropriate genotype, and upon genotyping, female offspring were used for assessment of follicular pool. All animal work was conducted according to protocols approved by the Animal Oversight Committee at Mount Sinai Hospital.

Ovarian histomorphometry. Ovaries were removed from offspring (approximately 3 weeks old; days 24-26) of control and treated groups, fixed in Dietrich's fixative or buffered formalin phosphate, and embed- 
ded in paraffin. Whole ovarian tissue was serially sectioned at $5 \mu \mathrm{m}$ thickness, mounted on slides, and stained with hematoxylin and picric acid methyl blue stain. The total number of nonapoptotic primordial and primary follicles per whole ovary was determined in every tenth section, as previously described (44).

Ovarian in vitro culture. Postnatal day 4 neonatal ovaries were collected from ICR females and were cultured in serum-free conditions, as previously described (45), for 24 or 48 hours with vehicle (DMSO) or a mixture of DMBA/BaP $(1 \mu \mathrm{M} / 1 \mu \mathrm{M})$, resveratrol $(2 \mu \mathrm{M})$, or $\alpha-\mathrm{NF}(2 \mu \mathrm{M})$, all from Sigma-Aldrich. After this period, tissue was either fixed for immunohistochemical analysis or used for RNA or western blot expression studies. For experiments involving Hrk- and A $h r$-deficient females, ovaries were collected from all females born from heterozygote crosses. One ovary from each female was used as a vehicle-treated control and the other as tissue exposed to PAH for 24 hours. After genotyping, wild-type, Hrk-, or Abr-deficient ovarian pairs were subjected to gene expression analysis.

Human ovarian xenografts. Human ovarian tissue was collected from 2 patients (ages 33 and 34) undergoing gynecological surgery (ovariectomy) for benign conditions, after patient informed consent was obtained following approval by the University of Toronto Research Ethics Committee. Tissue from each patient was divided into 4 groups and treated as an independent experiment. Parts of the ovarian cortex were collected, cut into small, thin strips (approximately $2 \mathrm{~mm}^{3}$ ), and subcutaneously transplanted into at least 3 NOD/SCID mice per group, with at least 2 grafts per mouse. The transplant procedure and treatment were performed as previously described $(16,46)$. Mice carrying xenograft were exposed to vehicle (paraffin oil) or a single dose of DMBA $(50 \mathrm{mg} / \mathrm{kg})$ and/or cotreated with resveratrol $(50 \mathrm{mg} / \mathrm{kg})$ dissolved in propylene glycol. Ovarian tissue was collected 48 hours later, fixed in formalin, and subjected to immunohistochemical analysis for HRK expression.

Real-time RT-PCR. Total RNA was extracted from individual neonatal ovaries treated with various agents using TRIzOL (Invitrogen) supplemented with glycogen (Boehringer Manheim) and cleaned with PCRquality DNase enzyme (Sigma-Aldrich) according to the manufacturer's instructions. Complementary DNA was generated using First Strand Revert Aid kit (Fermentas). Hrk expression was monitored using quantitative RT-PCR using Assays-on-Demand Taqman primers and probe (Applied Biosystems), while PCR for $\beta$-actin was performed using the SYBR Green I dye DyNamo HS kit (MJ Research) based on the manufacturer's protocol using specific primers (BA-F: $5^{\prime}$-CCACAGCTGAGAGGGAAATC-3'; BA-R: 5'-TCCATACCCAAGAAGGAAGG-3'). Analysis was done using the DNA Engine Opticon 2 System (MJ Research), and data for all quantitative PCR analyses were normalized against expression of $\beta$-actin. In all experiments, vehicle-exposed ovaries were used for generation of a baseline value with which other treatment groups were compared. When genetically engineered mice were used, one ovary from the same animal was used for control and the other for treatment group, and individual pairs were then compared.

Western blotting. Approximately 3 -week-old female offspring as well as dams were sacrificed by cervical dislocation. Lung tissue was quickly excised, snap-frozen on dry ice, and stored at $-80^{\circ} \mathrm{C}$ until further processing. Ovaries from 4-day-old neonates were subjected to in vitro PAH exposure as described above. Frozen tissues were homogenized in icecold RIPA buffer. Protein concentrations were determined following the Pierce Biotechnology BCA Protein Assay Kit, and $40 \mu \mathrm{g}$ of protein was run on a $10 \%$ polyacrylamide gel and electrophoretically transferred onto nitrocellulose membrane. The membrane was blocked with $5 \%$ nonfat dry milk and incubated with a 1:100 concentration of goat polyclonal antibody against mouse CYP1A1, anti-mouse Hrk (Q17), or goat polyclonal antibody against $\beta$-actin (all from Santa Cruz Bio- technology Inc.), followed by anti-mouse or anti-goat immunoglobulin conjugated to HRP $(1: 1,500)$. Blots were exposed to chemiluminescent ECL-plus reagent (Amersham). All blots were confirmed for equal protein loading, adjusted for expression of $\beta$-actin, and quantitated using ImageQuant software.

Immunohistochemistry. Collected ovarian tissue was fixed in $10 \%$ buffered formalin phosphate and processed for paraffin-embedded sections. After endogenous peroxidase quenching in $1 \% \mathrm{H}_{2} \mathrm{O}_{2}$ and high-temperature (microwave) antigen retrieval in $10 \mathrm{mM}$ citrate buffer for Bax or $2 \mathrm{~N} \mathrm{HCl}$, treatment for Hrk was performed. Sections were incubated with either rabbit anti-Bax (P19), anti-Hrk polyclonal antibody (Q17) or goat anti-human HRK polyclonal antibody (N20), all from Santa Cruz Biotechnology Inc., overnight at $4^{\circ} \mathrm{C}$. Subsequent antibody detection was carried out using appropriate secondary biotinylated $\operatorname{IgG}$ antibody and Vectastain ABC kit (DAKO) with diaminobenzidine as peroxidase substrate (Sigma-Aldrich). Immunolocalization of DNA adducts was performed using 5D11 monoclonal antibody (kindly provided by Regina Santella, Columbia University, New York, New York, USA; 1:25) and MOM immunodetection kit (Vector Laboratories). This antibody recognizes BPDE-I-DNA adducts and also cross-reacts with diol epoxide DNA adducts of other PAHs (20). As a negative control, the primary antibody was omitted, but all remaining steps were performed. All sections were counterstained with hematoxylin, dehydrated, and mounted in DPX mounting medium.

Hrk promoter analysis and site-directed mutagenesis. The mouse Hrk promoter luciferase construct was generated from Hrk genomic clone obtained previously (37). 5.3-kb genomic fragment upstream of exon 1 was cloned into $\mathrm{KpnI} / \mathrm{XhoI}$ sites in pGL2 luciferase reporter vector (Promega Corp.). Site-directed mutagenesis of putative XRE sites in the mouse Hrk promoter was performed by PCR using the QuikChange XL Site-Directed Mutagenesis Kit (Stratagene). Primers containing oligonucleotide substitutions of the first 2 base pairs of all 3 XREs to adenines $(\mathrm{CGTG} \rightarrow \mathrm{AATG}$ ) were used to generate inactive XREs and are listed in Supplemental Table 1. Mutagenesis of XRE nucleotides were confirmed by DNA sequence analysis. In addition, the inactive $\Delta 70 \mathrm{Hrk}$ promoter, missing 70 bp before exon 1 on pGL2 backbone, was used as a background control. HEK293 cells grown in DMEM supplemented with glutamine, nonessential amino acids, and $10 \%$ of FCS in 24-well plates were transiently transfected with either Hrk-WT, Hrk- 3 XRE mutated, or $\Delta 70$ $H r k$ constructs in concentrations of $0.55 \mu \mathrm{g}$ of plasmid per well using the $\mathrm{CaCl}_{2}$ method (47). Twenty-four hours after transfection, cells were treated with vehicle (DMSO) or PAHs ( $1 \mu \mathrm{M} \mathrm{DMBA} / \mathrm{BaP}$ each) and harvested 24 hours later using reporter lysis buffer (Promega). Luciferase activity was measured using a luminometer (MicroLumat Plus LB96V; EG\&G Berthold). Luciferase activity was normalized to $\beta$-galactosidase activity resulting from cotransfection with $H r k$ reporter vectors at a concentration of $0.2 \mu \mathrm{g}$ per well to account for minor differences in the transfection efficiency. Normalized data are expressed as the mean fold change \pm SEM relative to the vehicle-treated wild-type Hrk promotertransfected cells. All experiments were performed in pentuplicate, and each experiment was repeated 3 times.

Statistics. Significance of morphometric analysis of the ovarian pool among different treatment groups was assessed by 1-way ANOVA, followed by Duncan's or Student-Newman-Keuls post-hoc test. The remaining data, when only 2 groups were compared, were subjected to Student's $t$ test. All tests were performed using the SPSS statistical package (version 11.0), and a $P$ value of less than 0.05 was considered significant.

\section{Acknowledgments}

The authors would like to thank Paul Gerczuk for help with ovarian morphometry, Theodore Brown for the generous gift of 
$\beta$-galactosidase plasmid, and Alexandra Kollara for advice during the course of this study. We would also like to thank Jonathan Tilly for providing us with Abr genetically engineered mice and Santella for the BPDE antibody. This work was supported by research grants from the Banting and Best Research Foundation, and from the Canadian Institute of Health Research (MOP 200209 and FRN 63627 to A. Jurisicova).

1. Sharara, F.I., Seifer, D.B., and Flaws, J.A. 1998 Environmental toxicants and female reproduction. Fertil. Steril. 70:613-622.

2. Sram, R. 1999. Impact of air pollution on reproductive health. Environ. Health Perspect. 107:A542-A543.

3. Tilly, J.L. 1998. Molecular and genetic basis of normal and toxicant-induced apoptosis in female germ cells. Toxicol. Lett. 102-103:497-501.

4. Practice Committee of the American Society for Reproductive Medicine. 2004. Smoking and infertility. Fertil. Steril. 82(Suppl. 1):S62-S67.

5. Billaud, N., and Lemarie, P. 2001. Negative effects of maternal smoking during the course of pregnancy [In French]. Arch. Pediatr. 8:875-881.

6. Harlow, B.L., and Signorello, L.B. 2000. Factors associated with early menopause. Maturitas. 35:3-9.

7. Thomford, P.J., and Mattison, D.R. 1986. The effect of cigarette smoking on female reproduction. J. Ark. Med. Soc. 82:597-604.

8. Mattison, D.R., Shiromizu, K., and Nightingale, M.S. 1983. Oocyte destruction by polycyclic aromatic hydrocarbons. Am. J. Ind. Med. 4:191-202.

9. Mattison, D.R., Singh, H., Takizawa, K., and Thomford, P.J. 1989. Ovarian toxicity of benzo(a)pyrene and metabolites in mice. Reprod. Toxicol. 3:115-125.

10. Vahakangas, K., Rajaniemi, H., and Pelkonen, O. 1985. Ovarian toxicity of cigarette smoke exposure during pregnancy in mice. Toxicol. Lett. 25:75-80.

11. Matikainen, T.M., et al. 2002. Ligand activation of the aromatic hydrocarbon receptor transcription factor drives Bax-dependent apoptosis in developing fetal ovarian germ cells. Endocrinology. 143:615-620.

12. Weinberg, C.R., Wilcox, A.J., and Baird, D.D. 1989. Reduced fecundability in women with prenatal exposure to cigarette smoking. Am. J. Epidemiol. 129:1072-1078.

13. Denissenko, M.F., Venkatachalam, S., Ma, Y.H., and Wani, A.A. 1996. Site-specific induction and repair of benzo[a]pyrene diol epoxide DNA damage in human $\mathrm{H}$-ras protooncogene as revealed by restriction cleavage inhibition. Mutat. Res. 363:27-42.

14. Denison, M.S., and Nagy, S.R. 2003. Activation of the aryl hydrocarbon receptor by structurally diverse exogenous and endogenous chemicals. Annu. Rev. Pharmacol. Toxicol. 43:309-334.

15. Hankinson, O. 1995. The aryl hydrocarbon receptor complex. Annu. Rev. Pharmacol. Toxicol. 35:307-340.

16. Matikainen, T., et al. 2001. Aromatic hydrocarbon receptor-driven Bax gene expression is required for premature ovarian failure caused by biohazardous environmental chemicals. Nat. Genet. 28:355-360.

17. Zedeck, M.S. 1980. Polycyclic aromatic hydrocarbons: a review. J. Environ. Pathol. Toxicol. 3:537-567.

18. Casper, R.F., et al. 1999. Resveratrol has antagonist activity on the aryl hydrocarbon receptor: implica-
Received for publication March 13, 2006, and accepted in revised form September 21, 2007.

Address correspondence to: Andrea Jurisicova, Samuel Lunenfeld Research Institute, Room 876, Mount Sinai Hospital, 600 University Avenue, Toronto, Ontario M5G 1X5, Canada. Phone: (416) 586-4800, ext. 2052; Fax: (416) 586-8588; E-mail: jurisicova@mshri.on.ca. tions for prevention of dioxin toxicity. Mol. Pharmacol. 56:784-790.

19. Roos, W.P., and Kaina, B. 2006. DNA damageinduced cell death by apoptosis. Trends Mol. Med. 12:440-450.

20. Santella, R.M., Lin, C.D., Cleveland, W.L., and Weinstein, I.B. 1984. Monoclonal antibodies to DNA modified by a benzo[a]pyrene diol epoxide. Carcinogenesis. 5:373-377.

21. Inohara, N., Ding, L., Chen, S., and Nunez, G. 1997. Harakiri, a novel regulator of cell death, encodes a protein that activates apoptosis and interacts selectively with survival-promoting proteins $\mathrm{Bcl}-2$ and Bcl-X(L). ЕМBO J. 16:1686-1694.

22. Fisher, M.T., Nagarkatti, M., and Nagarkatti, P.S 2004. Combined screening of thymocytes using apoptosis-specific cDNA array and promoter analysis yields novel gene targets mediating TCDDinduced toxicity. Toxicol. Sci. 78:116-124.

23. Park, J.H., and Lee, S.W. 2002. Up-regulated expression of genes encoding Hrk and IL-3R beta subunit by TCDD in vivo and in vitro. Toxicol. Lett. 129:1-11.

24. Johnson, J., et al. 2005. Oocyte generation in adult mammalian ovaries by putative germ cells in bone marrow and peripheral blood. Cell. 122:303-315.

25. Johnson, J., Canning, J., Kaneko, T., Pru, J.K., and Tilly, J.L. 2004. Germline stem cells and follicular renewal in the postnatal mammalian ovary. Nature. 428: $145-150$

26. Ottolenghi, C., et al. 2004. Aging of oocyte, ovary, and human reproduction. Ann. N. Y. Acad. Sci. 1034:117-131.

27. Goswami, D., and Conway, G.S. 2005. Premature ovarian failure. Hum. Reprod. Update. 11:391-410.

28. Mattison, D.R. 1979. Difference in sensitivity of rat and mouse primordial oocytes to destruction by polycyclic aromatic hydrocarbons. Chem. Biol. Interact. 28:133-137.

29. Mattison, D.R., and Nightingale, M.S. 1982. Oocyte destruction by polycyclic aromatic hydrocarbons is not linked to the inducibility of ovarian aryl hydrocarbon (benzo(a)pyrene) hydroxylase activity in (DBA/2N X C57BL/6N) F1 X DBA/2N backcross mice. Pediatr. Pharmacol. (New York) 2:11-21.

30. Takizawa, K., Yagi, H., Jerina, D.M., and Mattison, D.R. 1984. Murine strain differences in ovotoxicity following intraovarian injection with benzo(a)pyrene (+)-(7R,8S)-oxide (-)-(7R,8R)-dihydrodiol, or (+)-(7R,8S)-diol-(9S,10R)-epoxide-2. Cancer Res. 44:2571-2576.

31. Willis, S.N., and Adams, J.M. 2005. Life in the balance: how $\mathrm{BH} 3$-only proteins induce apoptosis. Curr. Opin. Cell Biol. 17:617-625.

32. Imaizumi, K., et al. 1999. The cell death-promoting gene DP5, which interacts with the BCL2 family, is induced during neuronal apoptosis following exposure to amyloid beta protein. J. Biol. Chem.
274:7975-7981

33. Harris, C.A., and Johnson, E.M., Jr. 2001. BH3-only Bcl-2 family members are coordinately regulated by the JNK pathway and require Bax to induce apoptosis in neurons. J. Biol. Chem. 276:37754-37760.

34. Wakabayashi, T., Kosaka, J., and Hommura, S. 2002. Up-regulation of Hrk, a regulator of cell death, in retinal ganglion cells of axotomized rat retina. Neurosci. Lett. 318:77-80.

35. Sanz, C., et al. 2000. Specific and rapid induction of the proapoptotic protein Hrk after growth factor withdrawal in hematopoietic progenitor cells. Blood. 95:2742-2747.

36. Kalinec, G.M., et al. 2005. Pivotal role of Harakiri in the induction and prevention of gentamicininduced hearing loss. Proc. Natl. Acad. Sci. U. S. A. 102:16019-16024.

37. Imaizumi, K., et al. 2004. Critical role for DP5/ Harakiri, a Bcl-2 homology domain 3-only Bcl-2 family member, in axotomy-induced neuronal cell death. J. Neurosci. 24:3721-3725.

38. Coultas, L., et al. 2007. Hrk/DP5 contributes to the apoptosis of select neuronal populations but is dispensable for haematopoietic cell apoptosis. J. Cell Sci. 120:2044-2052.

39. Strasser, A. 2005. The role of BH3-only proteins in the immune system. Nat. Rev. Immunol. 5:189-200.

40. Puthalakath, H., and Strasser, A. 2002. Keeping killers on a tight leash: transcriptional and posttranslational control of the pro-apoptotic activity of BH3-only proteins. Cell Death Differ. 9:505-512.

41. Coultas, L., et al. 2005. Concomitant loss of proapoptotic BH3-only Bcl-2 antagonists Bik and Bim arrests spermatogenesis. EMBOJ. 24:3963-3973.

42. DeKoning, E.P., and Karmaus, W. 2000. PCB exposure in utero and via breast milk. A review. J. Expo. Anal. Environ. Epidemiol. 10:285-293.

43. Robles, R., et al. 2000. The aryl hydrocarbon receptor, a basic helix-loop-helix transcription factor of the PAS gene family, is required for normal ovarian germ cell dynamics in the mouse. Endocrinology. 141:450-453.

44. Canning, J., Takai, Y., and Tilly, J.L. 2003. Evidence for genetic modifiers of ovarian follicular endowment and development from studies of five inbred mouse strains. Endocrinology. 144:9-12.

45. Eppig, J.J., and O’Brien, M.J. 1996. Development in vitro of mouse oocytes from primordial follicles. Biol. Reprod. 54:197-207.

46. Weissman, A., et al. 1999. Preliminary experience with subcutaneous human ovarian cortex transplantation in the NOD-SCID mouse. Biol. Reprod. 60:1462-1467.

47. Sambrook, J., and Russell, D.W. 2001. Molecular cloning: a laboratory manual. Cold Spring Harbor Laboratory Press. Cold Spring Harbor, New York, USA. 16.14-16.20. 